Physics in Collision (PIC 2013)

International Journal of Modern Physics: Conference Series

Vol. 31 (2014) 1460277 (11 pages)

(C) The Author

DOI: $10.1142 / \mathrm{S} 2010194514602774$

\title{
Top cross-sections and single top
}

\author{
Reinhard Schwienhorst \\ (On behalf of the ATLAS, CMS, CDF, D0 Collaborations) \\ Department of Physics \& Astronomy, \\ Michigan State University, 567 Wilson Road \\ East Lansing, Michigan 48823, USA \\ schwier@pa.msu.edu
}

Published 15 May 2014

\begin{abstract}
This paper summarizes top quark cross-section measurements at the Tevatron and the LHC. Top quark pair production cross-sections have been measured in all decay modes by the ATLAS and CMS collaborations at the LHC and by the CDF and D0 collaborations at the Tevatron. Single top quark production has been observed at both the Tevatron and the LHC. The $t$-channel and associated $W t$ production modes have been observed at the LHC and evidence for $s$-channel production has been reported by the Tevatron collaborations.
\end{abstract}

Keywords: Top quark; LHC; Tevatron.

\section{Introduction}

The top quark is central to understanding physics in the Standard Model (SM) and beyond. This paper summarizes top-quark related measurements from the Tevatron proton-antiproton collider at Fermilab and from the Large Hadron Collider (LHC), the proton-proton collider at CERN. The top quark couplings to the gluon, to the $W$ boson, and now also to the photon and $Z$ boson are all probed in these measurements. Searches for new physics in the top quark final state look for new particles and new interactions.

The Tevatron operation ended in 2011, with CDF and D0 each collecting $10 \mathrm{fb}^{-1}$ of proton-antiproton data ${ }^{1}$ at a center-of-mass (CM) energy of $1.96 \mathrm{TeV}$. The ATLAS $^{2}$ and $\mathrm{CMS}^{3}$ collaborations at the LHC have reported measurements at CM energies of $7 \mathrm{TeV}$ and $8 \mathrm{TeV}$, with up to $4.9 \mathrm{fb}^{-1}$ and up to $20 \mathrm{fb}^{-1}$, respectively.

This paper reports recent measurements of top pair production, of single top production, as well as recent searches for new physics in top quark final states. Giving a complete overview of all activities is not possible here, but I will highlight

This is an Open Access article published by World Scientific Publishing Company. It is distributed under the terms of the Creative Commons Attribution 3.0 (CC-BY) License. Further distribution of this work is permitted, provided the original work is properly cited. 


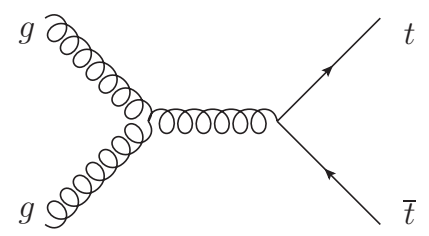

(a)

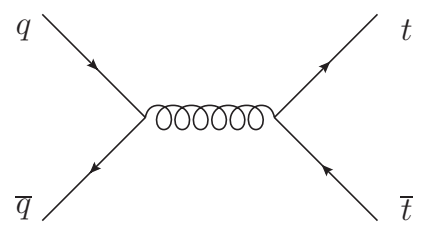

(b)

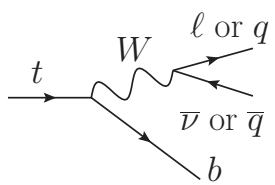

$(c)$

Fig. 1. Feynman diagrams for top pair production (a) via gluon fusion and (b) via quark-antiquark annihilation, and (c) for top quark decay.

relevant measurements and new developments. Section 2 summarizes top quark pair production measurements, Section 4 summarizes single top quark production, Section 5 presents new physics searches in the top quark sector, and Section 6 gives a summary.

\section{Top Quark Pair Production}

Top quark pair production proceeds mainly via gluon initial states at the LHC, shown in Fig. 1(a), and mainly via quark-antiquark annihilation at the Tevatron, shown in Fig. 1(b).

The production cross-section has been calculated at next-to-next-to leading order (NNLO), including next-to-next-to leading log (NNLL) soft gluon resummation. ${ }^{4}$

The top quark decays to a $W$ boson and a $b$ quark, and the final state topology in top quark pair events is determined by the subsequent decay of the two $W$ bosons, as shown in Fig. 1(c). About a third of top pairs decay to the lepton+jets final state where one $W$ boson decays to an electron or muon and the other to a quark pair. The background to this final state is mainly from $W+$ jets production and QCD multi-jet events where one quark jet is mis-identified as a lepton. This final state topology has reasonable statistics and a manageable background while also allowing for the reconstruction of the two top quarks.

A small fraction of about $6 \%$ of top pair events decay to the dilepton (ee, $e \mu$ and $\mu \mu$ ) final state, which has small backgrounds from $Z+$ jets and diboson production. This topology is attractive for its clean signature, though the individual top quarks can not be reconstructed directly due to the presence of two neutrinos.

About $46 \%$ of top pair events decay to an all-hadronic final state which is overwhelmed by a large QCD multi-jet background. Other top pair decays involve $\tau$ leptons, and in particular hadronic $\tau$ decays are of interest because they provide sensitivity to non-SM top decays. Leptonic $\tau$ decays are included in the lepton+jets and dilepton final states, though the lower lepton $p_{T}$ and the presence of additional neutrinos modifies the event kinematics. 


\subsection{Lepton+jets final state}

The lepton+jets final state (where the lepton is an electron or a muon) has backgrounds that can be controlled and higher event statistics than the dilepton final state. Cross-section measurements both at the Tevatron and the LHC by ATLAS ${ }^{5}$ and $\mathrm{CMS}^{6}$ rely on $b$-quark identification ( $b$-tagging) as well as multivariate analysis techniques to separate the top pair signal from the background sources, mainly $W+$ jets and QCD multi-jet production. The CMS analysis at $7 \mathrm{TeV}$ utilizes the secondary vertex mass to discriminate the top quark pair signal from the backgrounds. ${ }^{6}$ This distribution is shown in Fig. 2 for different jet- and $b$-tag multiplicities. The measured cross-section is $158.1 \pm 11.0 \mathrm{pb}$ for an uncertainty of only $7 \%$.

Differential cross-sections have also been measured in top pair production by ATLAS at $7 \mathrm{TeV}^{7}$ and by CMS at $7 \mathrm{TeV}^{8}$ and $8 \mathrm{TeV}{ }^{9,10}$ The differential crosssection measured by ATLAS at $7 \mathrm{TeV}$ as a function of the top pair transverse momentum is shown in Fig. 3 (left). The differential cross-section is normalized to the total cross-section, thus canceling many systematic uncertainties.

\subsection{Dilepton final state}

The dilepton final state (di-electron, di-muon and electron-muon) is clean with small backgrounds and small uncertainties, hence it provides high-precision measurements
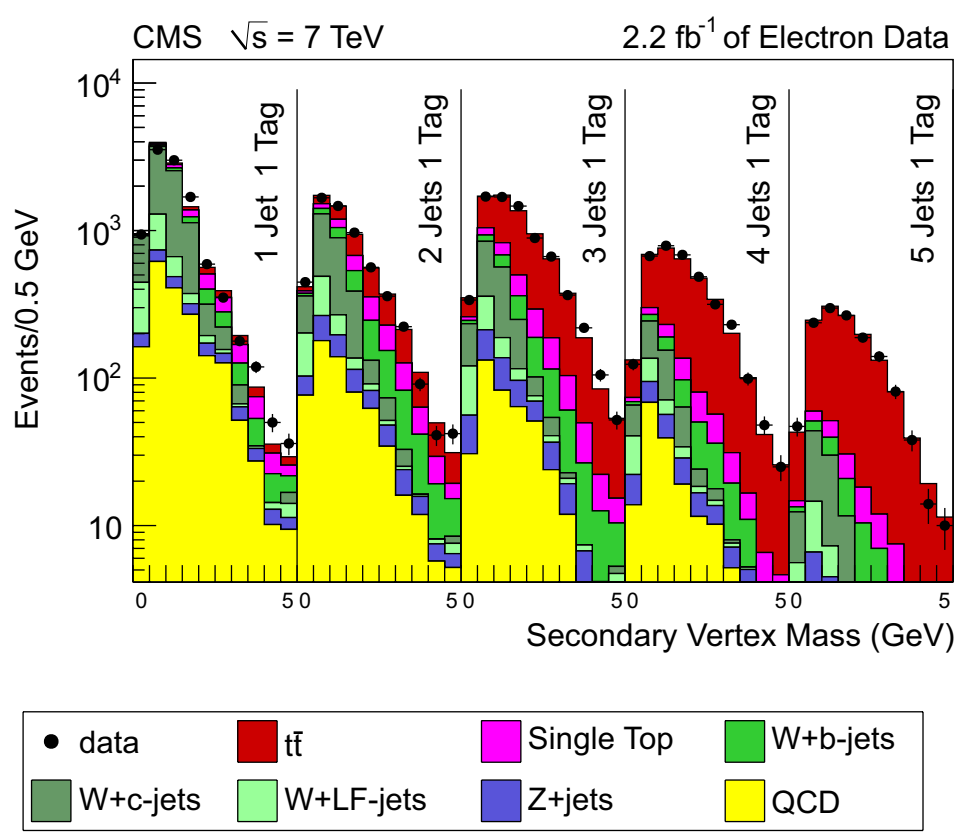

Fig. 2. Secondary vertex mass distribution in electron+jet events in the measurement of the top pair production cross-section at $7 \mathrm{TeV}$ by the CMS Collaboration. ${ }^{6}$ 

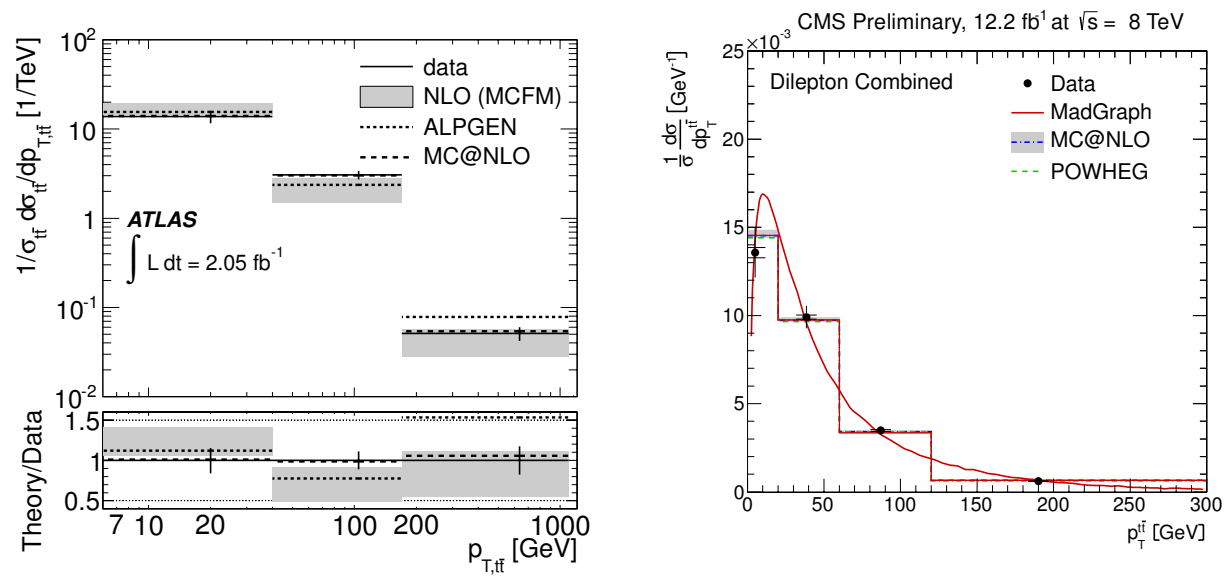

Fig. 3. (Left) ATLAS differential cross-section normalized to the total cross-section versus the transverse momentum of the $t \bar{t}$ system at $7 \mathrm{TeV} .{ }^{7}$ (Right) CMS differential cross-section normalized to the total cross-section versus the transverse momentum of the $t \bar{t}$ system at $8 \mathrm{TeV},{ }^{10}$ where data points are shifted horizontally to be directly comparable to the theory prediction.

of the production cross-section. The CMS measurement at $7 \mathrm{TeV}$ has an uncertainty of $4.2 \%$, currently the single most precise measurement. ${ }^{11}$

The differential cross-section has also been measured in the dilepton final state. Figure 3 (right) shows the relative differential cross-section of the transverse momentum of the top quark pair.

\subsection{Pair production summary}

The Tevatron measurements are summarized in Fig. 4. The combined Tevatron top pair cross-section is measured to be $7.60 \pm 0.41 \mathrm{pb}$, an uncertainty of only $5.4 \% .^{12}$

The measurements by ATLAS and CMS are shown as a function of the collider energy in Figs. 5 and 6, respectively. Note that the CMS summary figures do not yet include the latest CMS dilepton result. ${ }^{11}$ The measured cross-sections are consistent with each other and with the theory predictions. ${ }^{13,14}$ The LHC top pair cross-section combination from Fall $2012^{15}$ is also shown in Fig. 6.

\section{Associated Production}

Top quark pair production in association with one or more quarks or with a $W$ or $Z$ boson provides a measurement of the top quark strong and weak interactions and is an important background in new physics searches and Higgs boson measurements in $t \bar{t} H$.

The jet multiplicity in top pair events has been measured by both ATLAS ${ }^{18}$ and CMS. ${ }^{19,20}$ Figure 7 shows the jet multiplicity in lepton+jets top pair events. At low jet multiplicities, the measurement agrees within the large uncertainty band with the theoretical predictions, while at high jet multiplicities the agreement is poor. 


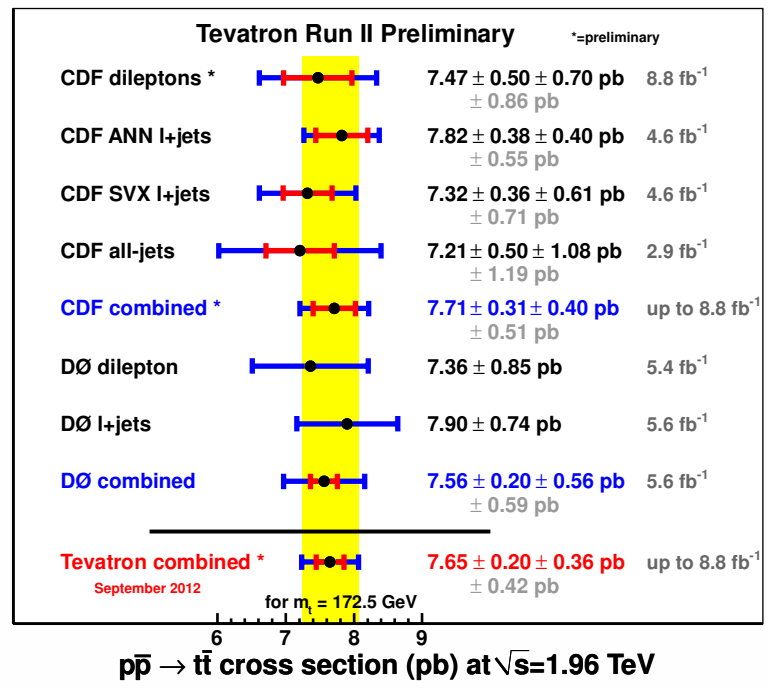

Fig. 4. Tevatron top quark pair production cross-section measurements and their combination. ${ }^{12}$

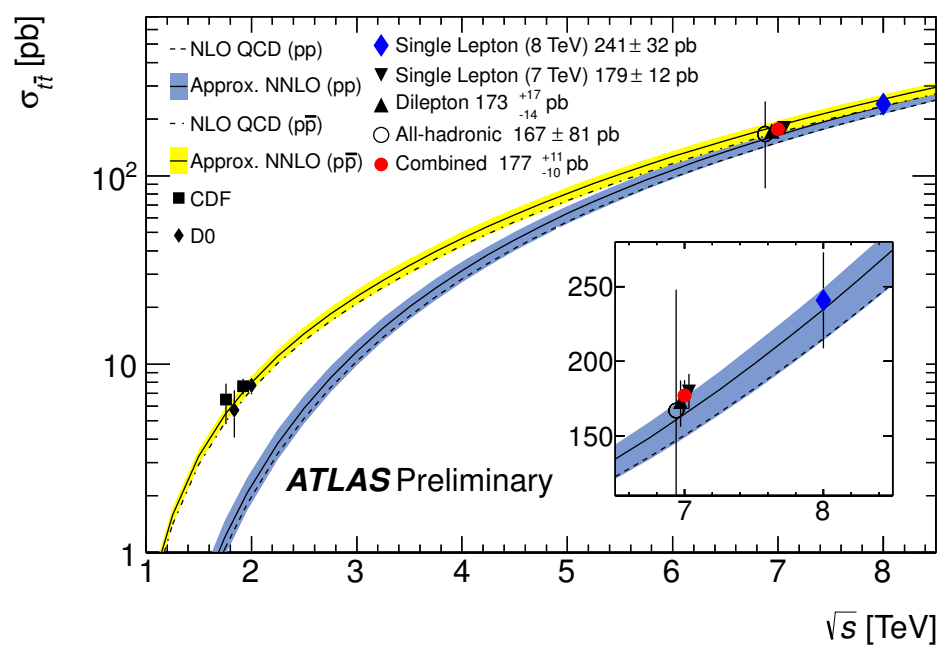

Fig. 5. ATLAS top quark pair production cross-section measurements as a function of collider energy. ${ }^{16}$

The production of top quark pairs in association with $Z$ bosons has a small crosssection and is difficult to measure. ATLAS had a first search for $t \bar{t} Z$ production at $7 \mathrm{TeV} .{ }^{22} \mathrm{CMS}$ found evidence for top pair production in association with a boson in two analyses ${ }^{21}$ using $7 \mathrm{TeV}$ data: A search for $t \bar{t} Z$ and a search for $t \bar{t} V$, where $V$ 


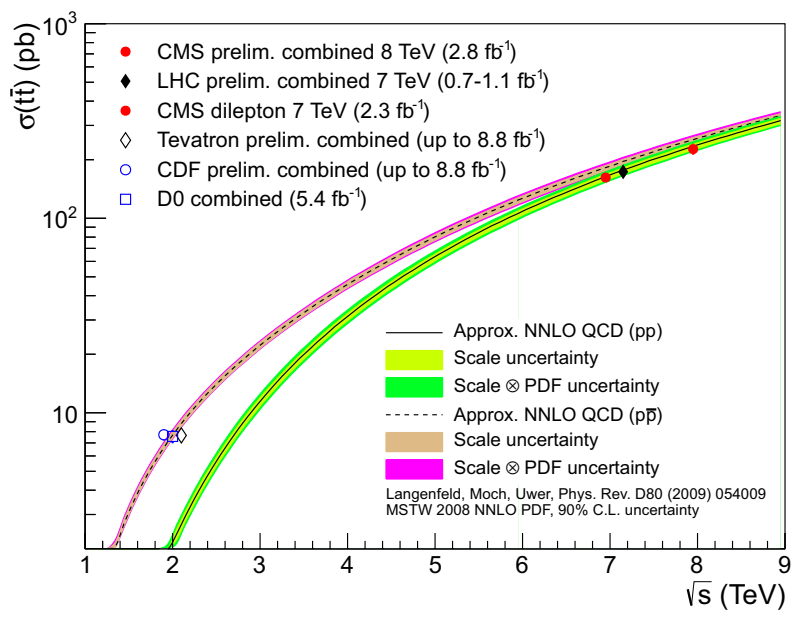

Fig. 6. CMS top quark pair production cross-section measurements as a function of collider energy. 17
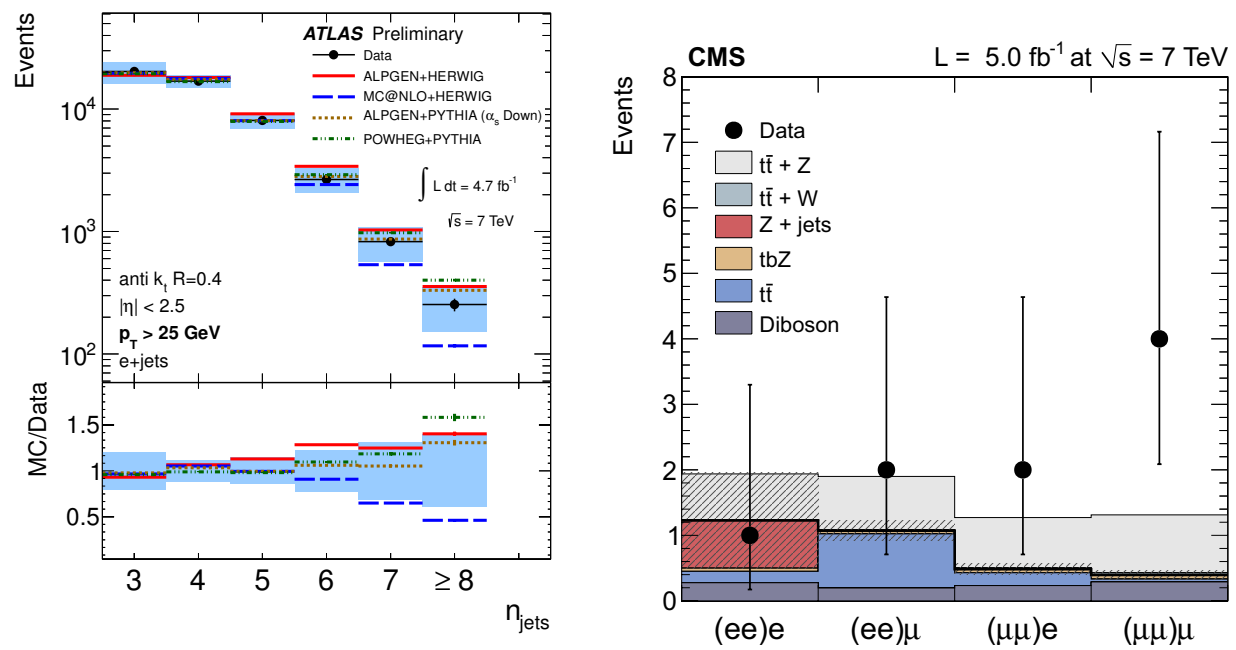

Fig. 7. Multiplicity of jets with $p_{T}>$ Fig. 8. Event yields in the tri-lepton channels in $25 \mathrm{GeV}$ in top quark pair events measured by the CMS $t \bar{t} V$ analysis at $7 \mathrm{TeV} .{ }^{21}$

ATLAS at $7 \mathrm{TeV} .^{18}$

can be a $W$ boson or a $Z$ boson. Figure 8 shows the two cross-section measurements and their uncertainties.

\section{Single Top Production}

Single top quark production proceeds via the $t$-channel exchange of a $W$ boson between a heavy quark line and a light quark line, shown in Fig. 9(a) or via the 


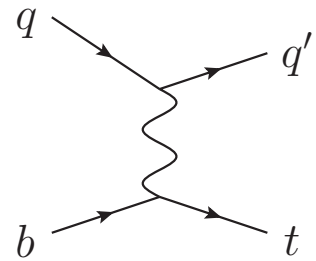

(a)

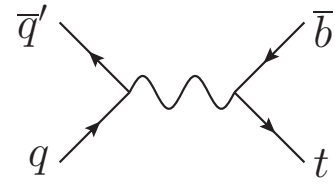

(b)

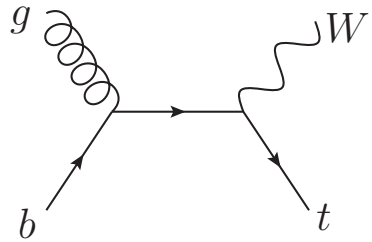

(c)

Fig. 9. Feynman diagrams for single top quark production in the (a) $t$-channel, (b) $s$-channel, (c) in association with a $W$ boson.

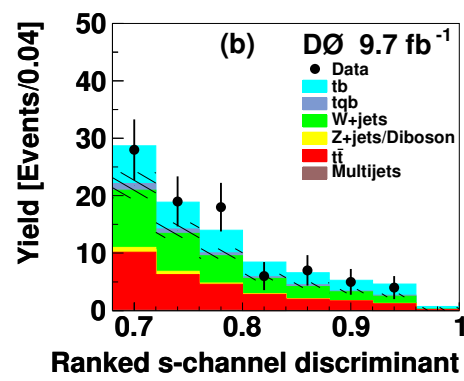

Fig. 10. D0 s-channel discriminant signal region. ${ }^{23}$

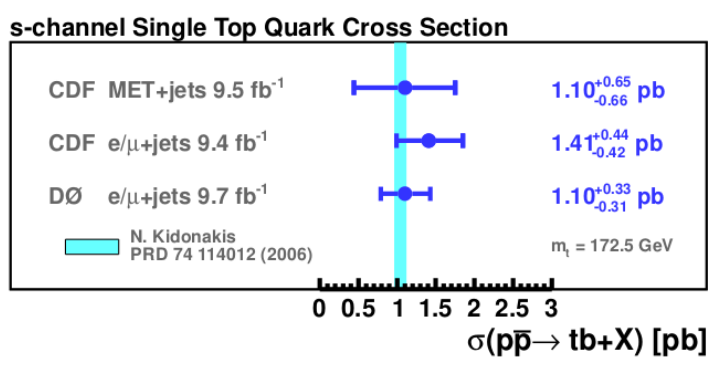

Fig. 11. Summary of Tevatron s-channel single top cross-section measurements.

$s$-channel production and decay of a virtual $W$ boson, shown in Fig. 9(b) or as the production of a top quark in association with a $W$ boson, shown in Fig. 9(c). At the Tevatron, the $t$-channel cross-section is largest, followed by the $s$-channel, while the $W t$ cross-section is too small to be observed. At the LHC, $t$-channel production benefits from the $q b$ initial state, with a large cross-section. The $s$-channel has a smaller cross-section and has not been seen yet. The associated production has a $g b$ initial state and can be observed.

\section{1. s-channel production}

Evidence for single top quark production in the $s$-channel was reported recently by the $\mathrm{D0}^{23}$ and $\mathrm{CDF}^{24}$ collaborations at the Tevatron. Both collaborations measure a cross-section that is consistent with the SM expectation, and both report an observed significance of 3.8 standard deviations. This is a challenging analysis that relies on multivariate analysis techniques in order to separate the signal from the large backgrounds. The $s$-channel signal region is shown in Fig. 10. A comparison of the CDF and D0 measurements is shown in Fig. 11. CDF also has a measurement using missing transverse energy plus jets events ${ }^{25}$ and a combination of the two results. ${ }^{26} \mathrm{~A}$ Tevatron combination of the CDF and D0 results is in progress. 


\subsection{Wt associated production}

The production cross-section of a single top quark in association with a $W$ boson has been measured both at $7 \mathrm{TeV}$ and at $8 \mathrm{TeV}$ by both ATLAS $^{27}$ and $\mathrm{CMS}^{28,29}$ The final state in $W t$ events is categorized by lepton multiplicity, similar to top pair production. The difference to top pair production is that $W t$ production results in exactly one high- $p_{T} b$-quark jet. Hence top pair events comprise the largest background in $W t$ dilepton events, with smaller contributions for $Z+$ jets, dibosons, and events with fake leptons. Multivariate techniques are required to separate the signal from these backgrounds, and systematic uncertainties are large. Nevertheless, the cross-section has now been measured with a relative uncertainty of $25 \%$. The $7 \mathrm{TeV}$ ATLAS analysis measures a cross-section of $16.8 \pm 2.9$ (stat) \pm 4.9 (syst) pb. ${ }^{27}$ The multivariate discriminant for the CMS $8 \mathrm{TeV}$ analysis is shown in Fig. 12, CMS cross-section measurement is $23.4 \pm 5.5 \mathrm{pb} .{ }^{29}$ Both are consistent with the SM expectation.

\section{3. t-channel production}

Single top quark production through the $t$-channel is sensitive to the parton distribution function (PDF) of the light quarks in the proton. The $t$-channel final state is comprised of a lepton, neutrino and $b$ quark from the top quark decay, a high- $p_{T}$ forward jet, and possibly a third jet. The main backgrounds to this signature are from $W+$ jets and top pair production. The ratio of top to antitop quark production in the $t$-channel is a particularly sensitive variable because many of the experimental uncertainties cancel. ATLAS has measured this ratio in $t$-channel events at $7 \mathrm{TeV}$ using a neural network to separate the $t$-channel events from the background. ${ }^{30}$ The measured ratio is $1.81_{-0.22}^{+0.23}$ and is compared to several different PDFs in Fig. 13.

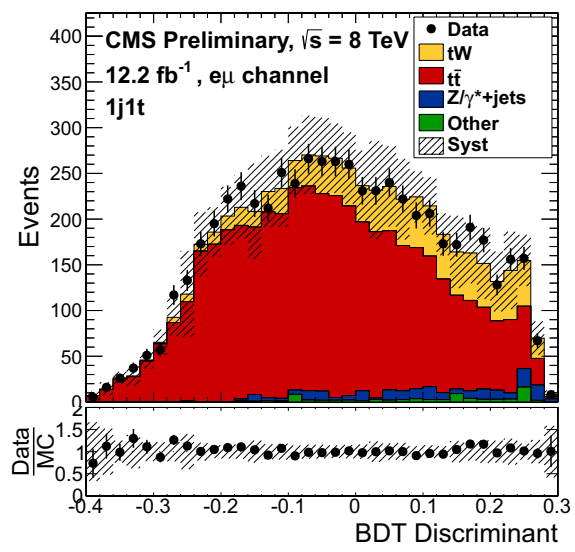

Fig. 12. CMS $W t$ discriminant in the $8 \mathrm{TeV}$ analysis. $^{29}$

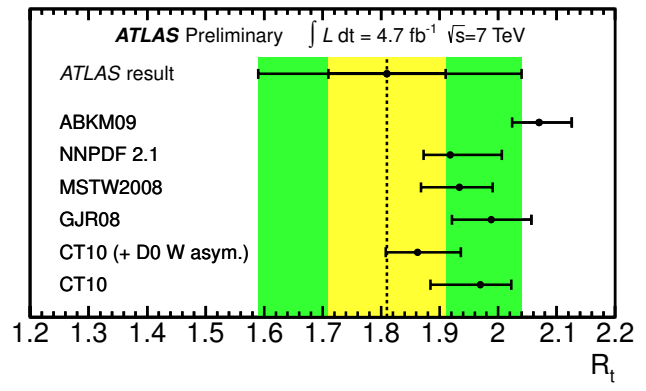

Fig. 13. Ratio of top to anti-top quark production in $t$-channel single top measured by ATLAS at $7 \mathrm{TeV}^{30}$ 
CMS has measured the same ratio using $8 \mathrm{TeV}$ data to be $1.76 \pm 0.27$, again consistent with expectations. ${ }^{31}$ The precision of these measurements is not yet sufficient to constrain PDFs, but future measurements should be able to improve on this situation. Along with the ratio, ATLAS and CMS have also measured the total $t$-channel cross section, with an uncertainty of $14 \%$ from ATLAS at $8 \mathrm{TeV}^{30}$ and an uncertainty of $9 \%$ from CMS at $7 \mathrm{TeV} .{ }^{32}$

\section{New Physics Searches}

Searches for new physics in the top quark sector are particularly sensitive to models that have an enhanced coupling to the third generation of fermions. New heavy bosons $Z^{\prime}$ and $W^{\prime}$ appear in many models of new physics, and searches for these heavy resonances are a priority at hadron colliders.

The reconstructed mass of the top quark pair system in the ATLAS $7 \mathrm{TeV}$ analysis is shown in Fig. $14 .^{33}$ The transverse momentum of the top quark produced in the decay of a heavy $Z^{\prime}$ boson is sufficient to collimate quark jets from the top quark decay such that they can no longer be resolved individually. Recent searches for $Z^{\prime}$ bosons employ algorithms to identify such boosted top quark jets. ${ }^{33,34}$ The $Z^{\prime}$ mass at which such algorithms perform better than resolved algorithms that reconstruct each of the top quark decay jets individually is around $1 \mathrm{TeV}$ as can be seen by the vertical dashed line in Fig. 15. The mass range probed by these searches extends up to masses of $2 \mathrm{TeV}$. The mass range below $0.75 \mathrm{TeV}$ is also probed at the Tevatron where a CDF search currently provides the best sensitivity. ${ }^{35}$

A new charged heavy boson $W^{\prime}$ can decay to a top quark together with a $b$ quark, leading to a single top final state. The $W^{\prime}$ boson may have SM-like left-handed couplings or it may have right-handed couplings to the top quark and the $b$ quark. The ATLAS ${ }^{36}$ and $\mathrm{CMS}^{37}$ analyses probe these couplings separately, with CMS also providing two-dimensional limits as a function of the two couplings.

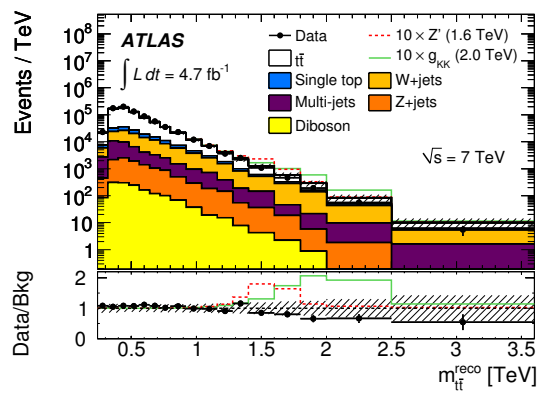

Fig. 14. Reconstructed invariant mass of the top quark pair system for the $7 \mathrm{TeV}$ ATLAS analysis. ${ }^{33}$

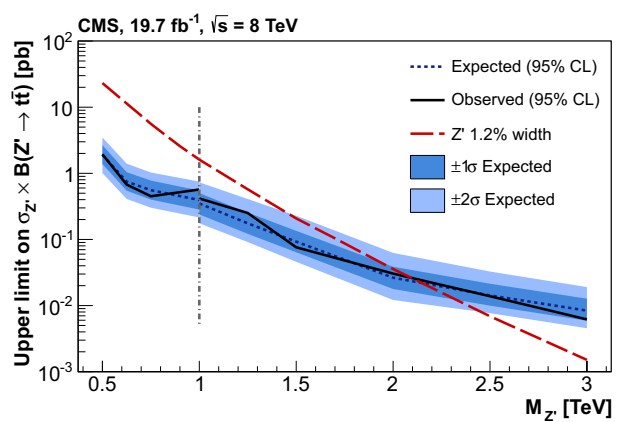

Fig. 15. Upper limit on $Z^{\prime} \rightarrow t \bar{t}$ from CMS at $8 \mathrm{TeV} .{ }^{34}$ 


\section{Summary}

The top quark pair production cross-section has been measured in many final states and with high precision by the CDF and D0 collaborations at the Tevatron protonantiproton collider and by the ATLAS and CMS collaborations at the $7 \mathrm{TeV}$ and $8 \mathrm{TeV}$ LHC proton-proton collider. The single top quark cross-sections have been measured in the $t$-channel and now also in the $s$-channel at the Tevatron, and in the $t$-channel and the $W t$ associated production at the LHC. Many of these measurements are now at the level of precision of the theory predictions, and higherprecision results are yet to come with $8 \mathrm{TeV}$ data. Searches for new physics in top quark final states have reached a sensitivity to high-mass resonances of over $2 \mathrm{TeV}$. This reach will be extended significantly at the $14 \mathrm{TeV}$ LHC.

\section{References}

1. D0 Collaboration, Nucl. Instrum. Meth. A565, 463 (2006).

2. ATLAS Collaboration, JINST 3, p. S08003 (2008).

3. CMS Collaboration, JINST 3, p. S08004 (2008).

4. M. Czakon, P. Fiedler and A. Mitov, Phys. Rev. Lett. 110, p. 252004 (2013).

5. ATLAS Collaboration, ATLAS-CONF-2012-149, http://cds.cern.ch/record/1493488.

6. CMS Collaboration, Phys. Lett. B720, 83 (2013).

7. ATLAS Collaboration, Eur. Phys. J. C73, p. 2261 (2013).

8. CMS Collaboration, Eur. Phys. J. C73, p. 2339 (2013).

9. CMS Collaboration, CMS-PAS-TOP-12-027, http://cds.cern.ch/record/1523611.

10. CMS Collaboration, CMS-PAS-TOP-12-028, http://cds.cern.ch/record/1523664.

11. CMS Collaboration, JHEP 1211, p. 067 (2012).

12. CDF Collaboration, D0 Collaboration, arXiv:1309.7570 (2013).

13. U. Langenfeld, S. Moch and P. Uwer, Phys. Rev. D80, p. 054009 (2009).

14. M. Aliev, H. Lacker, U. Langenfeld, S. Moch, P. Uwer et al., Comput. Phys. Commun. 182, 1034 (2011).

15. ATLAS Collaboration and CMS Collaboration, ATLAS-CONF-2012-134, CMS-PASTOP-12-003, http://cds.cern.ch/record/1541952.

16. ATLAS Collaboration (2012), http://twiki.cern.ch/twiki/bin/view/AtlasPublic/ CombinedSummaryPlots.

17. CMS Collaboration (2013), http://twiki.cern.ch/twiki/bin/view/CMSPublic/ PhysicsResultsTOPSummaryPlots.

18. ATLAS Collaboration, ATLAS-CONF-2012-155, http://cds.cern.ch/record/1493494.

19. CMS Collaboration, CMS-PAS-TOP-12-018, http://cds.cern.ch/record/1494576.

20. CMS Collaboration, CMS-PAS-TOP-12-041, http://cds.cern.ch/record/1547532.

21. CMS Collaboration, Phys. Rev. Lett. 110, p. 172002 (2013).

22. ATLAS Collaboration, ATLAS-CONF-2012-126, http://cds.cern.ch/record/1474643.

23. D0 Collaboration, Phys. Lett. B 726, p. 656 (2013).

24. CDF Collaboration, CDF note 11025, (2013).

25. CDF Collaboration, CDF note 11015, (2013).

26. CDF Collaboration, CDF note 11045, (2013).

27. ATLAS Collaboration, Phys. Lett. B716, 142 (2012).

28. CMS Collaboration, Phys. Rev. Lett. 110, p. 022003 (2013).

29. CMS Collaboration, CMS-PAS-TOP-12-040, http://cds.cern.ch/record/1563135. 
30. ATLAS Collaboration, ATLAS-CONF-2012-056, http://cdsweb.cern.ch/record/ 1453783.

31. CMS Collaboration, CMS-PAS-TOP-12-038, http://cds.cern.ch/record/1528574.

32. CMS Collaboration, JHEP 1212, p. 035 (2012).

33. ATLAS Collaboration, Phys. Rev. D88, p. 012004 (2013).

34. CMS Collaboration, Phys. Rev. Lett. 111, p. 211804 (2013).

35. CDF Collaboration, Phys. Rev. Lett. 110, p. 121802 (2013).

36. ATLAS Collaboration, ATLAS-CONF-2013-050, http://cds.cern.ch/record/1547566.

37. CMS Collaboration, CMS-PAS-B2G-12-010, http://cds.cern.ch/record/1525924. 